\section{ANTIBIOTIC RESISTANCE}

\section{Educate your colleagues}

Sir, antimicrobial resistance cannot be eradicated but a multi-disciplinary approach will minimise its impact. The UK Chief Medical Officer has voiced her concerns: 'Without action, there will come a point when regular surgery and other medical procedures carry a substantial risk of death.'

As NHS general practice dentists are responsible for nearly 10\% of all oral antimicrobial prescriptions in the primary care setting, we clearly have a role to play in reducing antibiotic resistance. GDC Standards require us to provide treatment based on 'current evidence and authoritative guidance' and when prescribing medicines only do so to meet 'identified dental need'. Antibiotics should only be prescribed as an adjunct to definitive treatment, where there are associated systemic symptoms such as an elevated temperature or local lymph gland involvement. NICE is currently developing guidance entitled Antimicrobial stewardship and antibiotic resistance changing risk-related behaviour.

Recognising the importance to newly qualified dentists, Health Education (North East) required its 2012/2013 Dental Foundation Trainees to audit antimicrobial prescribing in their training practices against FGDP(UK) guidance: Antimicrobial prescribing for general dental practitioners ( $2^{\text {nd }}$ edition, published 2012). In this letter, we refer to the results of the audit from 40 of these practices (some 2,500 prescriptions). As with previous audits, improvements were seen after the introduction of a prescribing protocol during the course of the audit. In summary:

- Whilst over 95\% of adult prescriptions met the dose criteria both before and after introduction of the FGDP-based prescribing protocol to dentists in the practice, only $66 \%$ of paediatric doses were correct before and 72\% after

- 71\% of amoxicillin prescriptions were correct for duration before and 95\% after; metronidazole prescription durations were correct in only 30\% of prescriptions before, increasing to only $74 \%$ after

- $98 \%$ of prescriptions were for amoxicillin or metronidazole; erythromycin was the third most prescribed antibiotic, yet was omitted from FGDP guidance due to the high level of side effects and bacterial resistance

- Particular improvements were seen in clinical indication for prescriptions; for example, 15 prescriptions were for irreversible pulpitis (which is never an appropriate indication) before compared to none after.

Notably, all of the incorrect doses were too low and all of the incorrect durations were too long; this is the worst combination as it produces the highest selective pressure for antimicrobial resistance. Colleagues are, therefore, encouraged to educate all in their practices about antimicrobial resistance and to audit prescribing against current guidelines.

With thanks to Health Education (North East), their 2012/13 DFTs and their Foundation Training Practices. Also, thanks to Christine Randall, North West Medicines Information Centre.

\section{W. Thompson, by email} DOI: 10.1038/sj.bdj.2014.713

\section{FITNESS TO PRACTISE}

\section{Unbelievably arrogant}

Sir, in February 2011 I started a simple course of upper arch orthodontic treatment for a patient. I was asked if I would give a discount for cash or if a discount could be applied, as the patient could obtain cheaper treatment in China (I declined both requests). All went well. A year later treatment was completed; the patient was happy with the result and had paid in full, without discount, prior to completion. On reviewing the upper retainer I was asked if I could provide a lower removable retainer. I agreed to do so for $£ 60$. The patient felt this should be included in the cost of orthodontic treatment, I explained why this was not the case (we had not agreed a treatment plan involving a lower retainer, only an upper one), the patient reminded me that it could be obtained at less cost in China. I agreed that was likely. The patient left and was discharged from my care.

Six months later I received a letter from the GDC stating that the patient had made a complaint. I had been accused of racist behaviours throughout the course of treatment. The GDC listed a charge of professional misconduct and a fitness to practise case was to be heard. I was asked to provide a list of my employers/places of work. The GDC immediately contacted my employers to inform them of the complaint and the reason for it; they also instructed them that a fitness to practise hearing was to be held. The GDC requested the patient record and trawled through it to see if they could find anything else to add to the charge list, whether related to the initial complaint or not.
Time moved on very slowly. I experienced sleepless nights and increasing levels of stress associated with the threat of losing a living. I had to take several days from clinic to attend meetings with Dental Protection and their legal team. I was asked to approach friends and acquaintances for character references. My legal team had to write to them and set out the list of charges before asking for the reference, all very embarrassing.

Two years following the initial complaint to the GDC I was instructed that the hearing was to be held in the last week of July 2014, I was told to cancel the week's clinics and book a hotel in London. Two months before the hearing I received notification that the complainant had refused to provide a witness statement and was refusing to attend the hearing. The GDC wrote advising that the "likelihood of securing the case as proven was low' and that with advice the hearing was cancelled.

As of yet they have not written to my employers informing them of the outcome and the reasons for it. When contacted, the GDC advised that informing employers of such an outcome is not something they would do. I asked Dental Protection what the approximate cost of this process would be; I was amazed to hear it would be $£ 15,000-20,000$.

I can conclude the following. The GDC is not fulfilling its role in a fair nor efficient way. Asking registrants for more money to fund such a system is unbelievably arrogant. Any patient can cause havoc without facing redress. Legal advice is expensive. The whole process is wrong.

S. Ward, by email

DOI: 10.1038/sj.bdj.2014.714

\section{FACIAL BURNS}

\section{Reducing risks}

Sir, we were interested to read the letter about a facial burn caused by acid etch (BDJ 2014; 217: 56). We congratulate the authors on bringing this adverse incident to the attention of the $B D J$ readership including how to manage the burn and suggestions to prevent similar episodes. We have two comments regarding this issue. 1. Care should always be taken not to pass any instruments or materials over the patient's face; and 2. for British Orthodontic Society members adverse events can be reported, confidentially, via the BOS website (www.bos.org.uk). Participation will help to reduce potential risks to patients undergoing orthodontic treatment.

R. Evans, London

D. Johnston, Ballymena DOI: 10.1038/sj.bdj.2014.715 